\title{
The Research Based on Big Data Management Accounting Model Building
}

\section{Zhang Hang, Liu Yun}

College of Wealth Management, Ningbo Dahongying University, Ningbo, China

\section{Email address:}

zhanghang119@sohu.com (Zhang Hang), ly2005112@163.com (Liu Yun)

\section{To cite this article:}

Zhang Hang, Liu Yun. The Research Based on Big Data Management Accounting Model Building. Science Innovation. Vol. 4, No. 4, 2016, pp. 228-234. doi: 10.11648/j.si.20160404.20

Received: September 12, 2016; Accepted: October 12, 2016; Published: November 2, 2016

\begin{abstract}
This paper is the study of a multivariate regression model construction related to management accounting,based on the nature of accounting under the viewpoint of the big data and cloud computing perspective. This paper chooses the multi-factor time series prediction construction model, bring in the steady test of time intervening variable, find outthe dependence relationship between independent variable, dependent variableand the time series variable dependence. It improves the simulation levelof forecast model building and the significance level of variable coefficient, providing reliable basis for prediction and decision-making. The final prediction model structurein this paper is the combination of a straight line and curve prediction model structure.
\end{abstract}

Keywords: Management Accounting, Prediction Model, Intermediary Variables, Hybrid Structure

\section{基于大数据管理会计预测模型构建的研究}

张航, 刘云

财富管理学院, 宁波大红鹰学院, 宁波, 中国

邮箱

zhanghang119@sohu.com（张航）, 1y2005112@163.com（刘云）

摘要：本文基于会计的自然属性在大数据，云计算视角下，对管理会计多元回归模型的构建进行研究，文中选择了时 间序列多因素构建预测模型，引入时间中介变量对模型进行稳健测试，分别找到了自变量、因变量与时间序列变量之 间的依存关系，使构建的预测模型的模拟水平和变量系数的显著性水平得到了提高，为预测、决策提供了可靠依据。 本文得出的最终预测模型结构是直线与曲线结合的混合模型结构。

关键词：管理会计, 预测模型, 中介变量, 混合结构

\section{1. 引言}

互联网、大数椐、云计算给我们带到了信息化时代, 会计专业也有了会计信息化学术术语, 会计理论与方法因 为互联网的信息化的迅速传递和会计业务的便捷化、电算 化处理, 也受之影响, 意义深远, 成为了历史发展过程的
必然。于是, 会计的理论在发生变化, 会计的属性也改为 了信息系统; 会计方法在发生变化, 其业务处理电算化, 其财务分析模型化; 会计的职能发生了变化, 由简单的会 计核算中的记账、算账, 到会计为中观、宏观预测、决 策……本文仅就会计相关学科《管理会计》之预测决策, 结合互联网数据, 应用计算机办公软件之Excel功能, 对 趋势分析的模型结构进行研究, 以期展示: “数据+计算 
机工具+管理会计+预测模型”（本文系作者实证会计方法 研究之阶段性研究成果), 理论与实践相结合的鬼力。本 文作者认为会计具有双重属性, 即具有社会属性, 还具有 自然属性, 管理会计与数学、统计学的方法联系是会计的 自然属性的有力验证, 本文的方法也是基于会计的自然属 性的展示。

在当今实证方法研究风靡, 会计的实证方法也不例外。 然, 综观相关学术期刊所刊的论文, 其模型结构多是直线 性, 而曲线型、混合型较少; 其显著性多是在直线性条件 下的正态分布 (二项式分布) 下的学生 $t$ 和p值检测, 而或 略了总体模拟水平, 当 $\mathrm{R}$ 方在非常小的情况下, 其按照模 型去做含量分析, 其意义之微乎其微。产生这种结果的主 要原因可能是, 一是作者没有找到解释因素; 二是找到了 解释因素, 作者没有找到解释因素对被解释因素的作用方 式, 也即模型的结构类型; 也可能以上两种情况都存在。 本文假设, 找到了因变量与自变量的关联因素, 然后在模 型的结构上探索趋势分析之回归分析模型, 通过适当的模 型结构, 使之总体模拟水平提高, 学生t水平显著, 使这 样的模型既可以作含量分析, 又可以作回归预测, 使实证 模型的确立与应用更具有现实意义。

\section{2. 二元线性模型结构研究}

\section{1. RD二元回归}

资料来源: 和讯数椐, 将资料转入Excel; 计算机工 具: MicrosoftOffice2007 Excel。

表1 RD余额与LFR表（亿元）。

\begin{tabular}{|c|c|c|c|c|}
\hline & A & B & $\mathrm{C}$ & D \\
\hline 1 & 年份 & 序列 & 存款余额 & 财政收入 \\
\hline 2 & 2015 & 28 & 34787 & 8549 \\
\hline 3 & 2014 & 27 & 30666.4 & 7521.7 \\
\hline 4 & 2013 & 26 & 28922. 97 & 6908.41 \\
\hline 5 & 2012 & 25 & 26406.8 & 6408.49 \\
\hline 6 & 2011 & 24 & 23470.25 & 5925 \\
\hline 7 & 2010 & 23 & 20612. 16 & 4895.41 \\
\hline 8 & 2009 & 22 & 17833. 44 & 4122.04 \\
\hline 9 & 2008 & 21 & 14504. 72 & 3730.06 \\
\hline 10 & 2007 & 20 & 11162. 82 & 3239.89 \\
\hline 11 & 2006 & 19 & 10473.5 & 2564.66 \\
\hline 12 & 2005 & 18 & 8746 & 2115.36 \\
\hline 13 & 2004 & 17 & 7364.1 & 1805.16 \\
\hline 14 & 2003 & 16 & 6452.2 & 1468.89 \\
\hline 15 & 2002 & 15 & 5212.7 & 1166.58 \\
\hline 16 & 2001 & 14 & 4262.38 & 917.76 \\
\hline 17 & 2000 & 13 & 3594.65 & 658.42 \\
\hline 18 & 1999 & 12 & 3262.7 & 477. 4 \\
\hline 19 & 1998 & 11 & 2847.29 & 401.8 \\
\hline 20 & 1997 & 10 & 2293.55 & 340.52 \\
\hline 21 & 1996 & 9 & 1844.74 & 291.75 \\
\hline 22 & 1995 & 8 & 1377.22 & 248.5 \\
\hline 23 & 1994 & 7 & 990.26 & 209. 39 \\
\hline 24 & 1993 & 6 & 665.64 & 166.64 \\
\hline 25 & 1992 & 5 & 514.44 & 118. 36 \\
\hline 26 & 1991 & 4 & 402. 09 & 108.94 \\
\hline 27 & 1990 & 3 & 306.74 & 101.59 \\
\hline 28 & 1989 & 2 & 214. 98 & 98.21 \\
\hline 29 & 1988 & 1 & 144. 03 & 85.55 \\
\hline
\end{tabular}

居民存款余额：浙江省1988-2015年城乡居民人民币 储蓄存款余额, 简写为RD; 财政收入: 浙江省1988-2015 年地方财政收入，简写为LFR。

重新排序, 以时间序号为自变量, 以存款余额为因变 量。选中新 “序号”与 “存款余额” 两列, 点击菜单 “插 入”、“散点”图, 选择曲线。有散点图形如下:

将图形粘贴到Word文档界面选中图, 右击键, “添加 趋势线” , 在跳出的界面选择 “线性” , 并勾选 “显示公 式”、“显示R方值” , 则存款的TSD直线性模型在界面显 示。由 $\mathrm{R}^{2}=0.8357$ 表明, 模拟水平不是不是很高的, 只有 $83.57 \%$ 。

重新选中图线, 右击键, 调出对话框, 点击 “添加趋 势线”，选 “二次项”，并勾选 “显示公式”、“显示R 方值” , “关闭” 以上图线就会出现一道模拟曲线, 并显 示模型公式和模拟水平。由 $\mathrm{R}^{2}=0.991$ 可知, 模拟水平很高, TSD对存款余额的作用呈二次项曲线。

重复以上方法, 在线性类型中选“三次项”, 其模拟水 平更高为 $\mathrm{R}^{2}=0.9962$, 模拟曲线几乎与实际线重合, 这时 可以用此公式对RD余额进行预测了。

2016年底浙江省城乡居民人民币存款为:

$184.03+1.8512 * 29^{\wedge} 3-9.4159 * 29^{\wedge} 2+78.021 * 29=31374.02$ ( 亿元 $)$

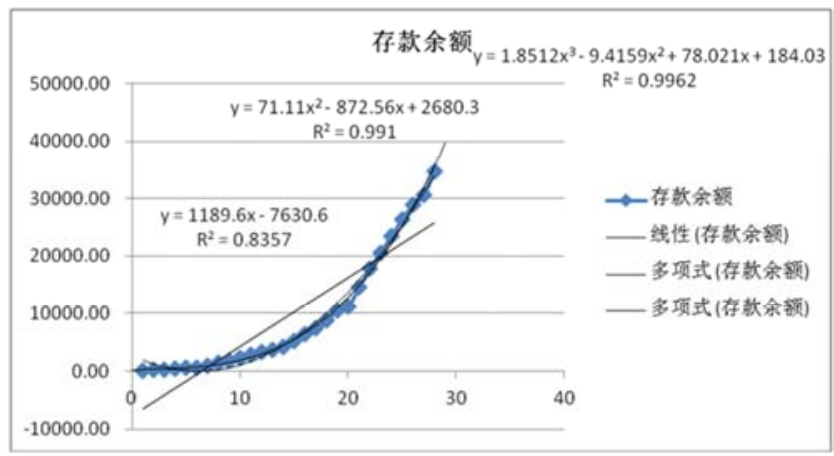

图1 RD\TSD趋势图。

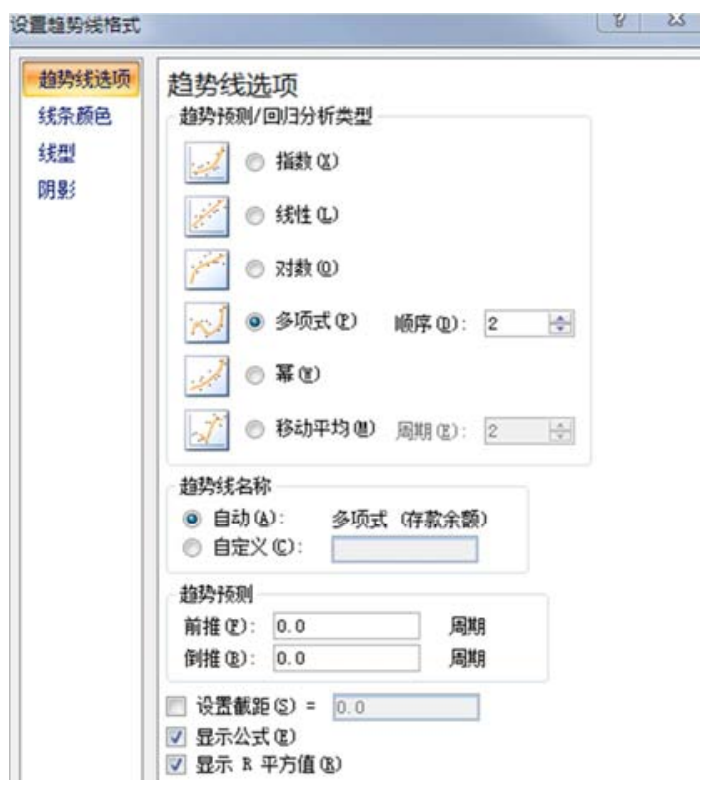

图2 趋势线类型。 


\section{2. LFR二元回归}

在Exce1界面把表1中的TSD调到 “LFR” 之前, 构成双 因素, 检测一下TSD对LFR影响关系式怎样？我们得到以下 图线: 其三次趋势线添加后, 各回归模型和模拟水平如图
所示, 模拟水平线性、二次项、三次项依次为 $R^{2}=0.8286$ 、 $\mathrm{R}^{2}=0.9949 、 \mathrm{R}^{2}=0.9972$

看来LFR与TSD的不同模型中, 三次项的模拟水平最高; 二次项与三次项的模拟曲线几乎与实际LFR曲线重合。

我们可以运用LFR与TSD的模型进行趋势分析, 或者叫 预测，2016年浙江省LFR预测为:

$\mathrm{Y}=0.3034 * 29^{\wedge} 3+5.019 * 29^{\wedge} 2-79.263 * 29+289.48=9900.93$ （亿元）。

2.3. 数据架构模拟水平比较

表2 RD、LFR与TSD不同模拟水平比较。

\begin{tabular}{|c|c|c|c|c|}
\hline & 线性回归 $\mathrm{R}^{2}$ & 二次项回归 $\mathrm{R}^{2}$ & 三次项回归 $\mathrm{R}^{2}$ & 评价 \\
\hline RD & 0.8357 & 0.991 & 0.9962 & \multirow{2}{*}{ 模拟水平逐步提高 } \\
\hline LFR & 0.8286 & 0.9949 & 0.9972 & \\
\hline
\end{tabular}

以上表明，二元回归中的TSD对RD、LFR作用方式呈二次项或三次项曲线关系。

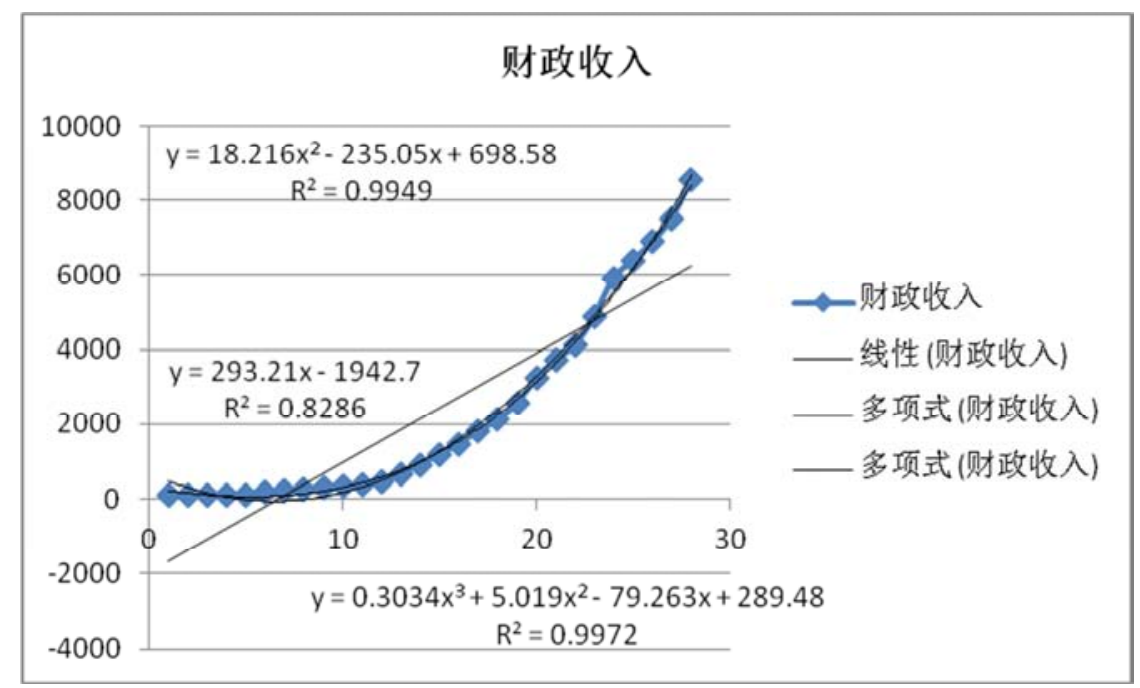

图3 LFR与TSD趋势图。

\section{MLR模型结构研究}

\section{1. 线性多元线性回归}

假设表1中, 我们用TSD与RD因素来解释它们对LFR的影响, 或者说把LFR的增长与RD的增长在TSD因素中是否有同步 发展, 或发展的协调性如何? 这是用Excel双因素由表到图, 再由图到模型公式, 就有些不太适应了。用MLR建立模型 时, Exce1仍然适用。可以在Exce1选项中调出“加载项”, 从“加载宏”中调出“数据分析”工具, 进而进行MLR分析和预测。 在没有对模型测试的基础上，我们用MLR的结果如下:

表3 回归统计。

\begin{tabular}{ll}
\hline MultipleR & 0.998081 \\
RSquare & 0.996165 \\
Ad justedRSquare & 0.995858 \\
标准误差 & 170.5274 \\
观测值 & 28 \\
\hline
\end{tabular}

表4 方差分析。

\begin{tabular}{llllll}
\hline & $\mathrm{df}$ & SS & MS & F & SignificanceF \\
\hline 回归分析 & 2 & $1.89 \mathrm{E}+08$ & 94416841 & 3246.841 & $6.27 \mathrm{E}-31$ \\
残差 & 25 & 726990.1 & 29079.6 & & \\
总计 & 27 & $1.9 \mathrm{E}+08$ & & & \\
\hline
\end{tabular}


表5 回归系数。

\begin{tabular}{lllllllll}
\hline & CoefficienTSD & 标准误差 & tStat & P-value & Lower95\% & Upper95\% & 下限95.0\% & 上限95.0\% \\
\hline Intercept & -35.5358 & 87.83768 & -0.40456 & 0.68924 & -216.441 & 145.3693 & -216.441 & 145.3693 \\
XVariable1 & -4.1217 & 9.842013 & -0.41879 & 0.678948 & -24.3917 & 16.1483 & -24.3917 & 16.1483 \\
XVariable2 & 0.249938 & 0.007563 & 33.04769 & $3.77 \mathrm{E}-22$ & 0.234361 & 0.265514 & 0.234361 & 0.265514 \\
\hline
\end{tabular}

以上TSD变量的学生 $\mathrm{t}$ 检测没有达到显著性要求, 说明TSD对财政的收入作用方式不是直线性的。基于双因素检测中, TSD对LFR的作用呈三项式模式, 所以我们引入曲线函数, 计算出TSD二次项、三次项的变量, 将曲线转化为直线性, 使 之可以用Exce1中的加载宏的数据分析作出改良的直线模型, 这种改良就是曲线部分转化为直线性模型。

\section{2. TSD二次项MLR}

表6 TSD曲线转化直线性。

\begin{tabular}{|c|c|c|c|c|c|}
\hline & $\mathrm{B}$ & $\mathrm{C}$ & $\mathrm{D}$ & $E$ & $\mathrm{~F}$ \\
\hline 1 & 序列 & 时间序列二次项 & 时间序列三次项 & 存款余额 & 财政收入 \\
\hline 2 & 28 & 784 & 21952 & 34787.00 & 8549.00 \\
\hline 3 & 27 & 729 & 19683 & 30666.40 & 7521.70 \\
\hline 4 & 26 & 676 & 17576 & 28922.97 & 6908.41 \\
\hline 5 & 25 & 625 & 15625 & 26406.80 & 6408.49 \\
\hline 6 & 24 & 576 & 13824 & 23470.25 & 5925.00 \\
\hline 7 & 23 & 529 & 12167 & 20612. 16 & 4895. 41 \\
\hline 8 & 22 & 484 & 10648 & 17833.44 & 4122.04 \\
\hline 9 & 21 & 441 & 9261 & 14504.72 & 3730.06 \\
\hline 10 & 20 & 400 & 8000 & 11162.82 & 3239.89 \\
\hline 11 & 19 & 361 & 6859 & 10473.50 & 2564.66 \\
\hline 12 & 18 & 324 & 5832 & 8746.00 & 2115. 36 \\
\hline 13 & 17 & 289 & 4913 & 7364.10 & 1805.16 \\
\hline 14 & 16 & 256 & 4096 & 6452.20 & 1468.89 \\
\hline 15 & 15 & 225 & 3375 & 5212.70 & 1166.58 \\
\hline 16 & 14 & 196 & 2744 & 4262.38 & 917.76 \\
\hline 17 & 13 & 169 & 2197 & 3594.65 & 658.42 \\
\hline 18 & 12 & 144 & 1728 & 3262.70 & 477. 40 \\
\hline 19 & 11 & 121 & 1331 & 2847.29 & 401.80 \\
\hline 20 & 10 & 100 & 1000 & 2293.55 & 340.52 \\
\hline 21 & 9 & 81 & 729 & 1844.74 & 291.75 \\
\hline 22 & 8 & 64 & 512 & 1377. 22 & 248.50 \\
\hline 23 & 7 & 49 & 343 & 990.26 & 209. 39 \\
\hline 24 & 6 & 36 & 216 & 665.64 & 166.64 \\
\hline 25 & 5 & 25 & 125 & 514.44 & 118. 36 \\
\hline 26 & 4 & 16 & 64 & 402. 09 & 108.94 \\
\hline 27 & 3 & 9 & 27 & 306. 74 & 101.59 \\
\hline 28 & 2 & 4 & 8 & 214.98 & 98.21 \\
\hline 29 & 1 & 1 & 1 & 144.03 & 85.55 \\
\hline
\end{tabular}

增加的TSD二次项、三次项如 (表6) 所示。根据转化了的数据, 然后用“数据分析”二次项回归结果如下:

表7 二次项MLR统计。

\begin{tabular}{ll}
\hline MultipleR & 0.998974 \\
RSquare & 0.997949 \\
AdjustedRSquare & 0.997692 \\
标准误差 & 127.2848 \\
观测值 & 28 \\
\hline
\end{tabular}

表8 三次项MLR统计。

\begin{tabular}{ll}
\hline MultipleR & 0.999018 \\
RSquare & 0.998037 \\
Ad justedRSquare & 0.997695 \\
标准误差 & 127.211 \\
观测值 & 28 \\
\hline
\end{tabular}

TSD二次项MLR的结果非常好

调整后的R方为0.997692（见表7）。回归系数的学生t检测都到达了99\%以上（见表8）。 
表9 二次项方差分析。

\begin{tabular}{llllll}
\hline & $\mathrm{df}$ & $\mathrm{SS}$ & MS & $\mathrm{F}$ & SignificanceF \\
回归分析 & 3 & $1.89 \mathrm{E}+08$ & 63057279 & 3892.082 & $2.23 \mathrm{E}-32$ \\
残差 & 24 & 388834.2 & 16201.43 & & \\
总计 & 27 & $1.9 \mathrm{E}+08$ & & & \\
\hline
\end{tabular}

二次项MLR结果如下:

表10 TSD二次项MLR结果。

\begin{tabular}{|c|c|c|c|c|c|c|c|c|}
\hline & CoefficienTSD & 标准误差 & tStat & $\mathrm{P}$-value & Lower95\% & Upper $95 \%$ & 下限95.0\% & 上限 $95.0 \%$ \\
\hline Intercept & 314.9928 & 100.923 & 3. 12112 & 0.004646 & 106. 698 & 523.2876 & 106. 698 & 523. 2876 \\
\hline XVariable1 & -110.173 & 24. 34786 & -4.5249 & 0.000139 & -160.425 & -59.9215 & -160.425 & -59.9215 \\
\hline XVariable2 & 8. 039134 & 1.759655 & 4. 56858 & 0.000124 & 4. 407386 & 11. 67088 & 4. 407386 & 11.67088 \\
\hline XVariable3 & 0.143112 & 0.024054 & 5. 94951 & 3. $86 \mathrm{E}-06$ & 0.093466 & 0.192758 & 0.093466 & 0.192758 \\
\hline
\end{tabular}

表11 TSD三次项方差分析。

\begin{tabular}{llllll}
\hline & $\mathrm{df}$ & SS & MS & F & SignificanceF \\
\hline 回归分析 & 4 & $1.89 \mathrm{E}+08$ & 47297118 & 2922.708 & $9.25 \mathrm{E}-31$ \\
残差 & 23 & 372200.6 & 16182.64 & & \\
总计 & 27 & $1.9 \mathrm{E}+08$ & & & \\
\hline
\end{tabular}

表12 TSD 三次项MLR结果。

\begin{tabular}{lllllllll}
\hline & CoefficienTSD & 标准误差 & tStat & P-value & Lower95\% & Upper95\% & 下限95.0\% & 上限95. 0\% \\
\hline Intercept & 268.4045 & 110.8389 & 2.421573 & 0.023745 & 39.11676 & 497.6922 & 39.11676 & 497.6922 \\
XVariable1 & -88.197 & 32.58812 & -2.70641 & 0.012594 & -155.611 & -20.7833 & -155.611 & -20.7833 \\
XVariable2 & 6.097213 & 2.60031 & 2.344802 & 0.028037 & 0.718062 & 11.47636 & 0.718062 & 11.47636 \\
XVariable3 & 0.091394 & 0.090147 & 1.013838 & 0.321208 & -0.09509 & 0.277876 & -0.09509 & 0.277876 \\
XVariable4 & 0.114513 & 0.037063 & 3.089633 & 0.005173 & 0.037841 & 0.191184 & 0.037841 & 0.191184 \\
\hline
\end{tabular}

表13 TSD、二次项、三次项MLR结果比较。

\begin{tabular}{|c|c|c|c|c|c|c|}
\hline & MLR & & 二次项MLR & & 三次项MLR & \\
\hline RSquare & 0.996165 & & 0.997949 & & 0.998037 & \\
\hline Ad justedRSquare & 0.995858 & & 0.997692 & & 0.997695 & \\
\hline \multirow[t]{6}{*}{ 显著性检测 } & tStat & P-value & tStat & P-value & tStat & P-value \\
\hline & -0.40456 & 0.68924 & 3. 12112 & 0.004646 & 2. 421573 & 0.023745 \\
\hline & -0.41879 & 0.678948 & -4.5249 & 0.000139 & -2.70641 & 0.012594 \\
\hline & 33. 04769 & 3. $77 \mathrm{E}-22$ & 4. 56858 & 0.000124 & 2. 344802 & 0.028037 \\
\hline & & & 5. 94951 & 3. $86 \mathrm{E}-06$ & 1. 013838 & 0.321208 \\
\hline & & & & & 3. 089633 & 0.005173 \\
\hline
\end{tabular}

由上表比较可知: MLR的总体模拟水平 $99.59 \%$, 但截 距项与 TSD的学生 $\mathrm{t}$ 检测都未通过显著性 $95 \%$ 的要求, 其分 别仅是 $31 \%$ 和 $32 \%$; 二次MLR的总体模拟水平高达 $99.77 \%$, 学生检测显著性也达 $5 \%$, 通过了显著水平 $99 \%$ 的检测; 三次项回归的总体模拟水平为 $99.77 \%$, 但其第三次项变量 的显著性水平仅为 $68 \%$, 没有通过 $95 \%$ 的统计显著性检验。 最佳回归结构模型为:

$$
y=314.9928-110.173 x_{1}+8.039134 x_{1}^{2}+0.143112 x_{2}
$$

\section{4. 结语}

综上所述, 我们可以得出以下结论:

\section{1 . 结果}

\section{1. 1. 资料层面}

互联网给我们提供了大数据。以上所用数据取之于 “和讯数据” 和浙江省2015年财政经济年报, 这似乎与经 济学所用数据指标相近, 其实, 互联网在证券之星、新浪 财经、网易财经、搜狐财经、东方财富、巨潮网、同花顺…… 为我们提供了大量的上市公司的财务数据, 这些都为我们 进行财务分析提供了数据资源。本文所取数据资料, 旨在 说明方法, 而对方法的应用予以忽略。上例中, 我们还可 以选择二次项多元回归模型, 设定浙江省居民存款预期, 来测算浙江省预期财政收入情况, 基于数据旨在说明方法, 
所以不再展开。若选择财务方面的指标进行企业经济效益 财务分析和财务预测, 同样适用。

\subsection{2. 工具方面}

本文所实施的回归模型数据处理软件, 并非 SolutionsStatisticalPackagefortheSocialSciences, 也非EconometricsViews、Matlab、STATA、SAS, 而是电 脑中Microsoft Office2007 Exce1，其2003、2007、2010 版本都带有加载宏数据分析功能 (WPS、内存小的尝鲜版 没有), 这为有效地进行财务回归分析和趋势预测提供了 便利的工具。

\subsection{3. 技术层面}

(1)数据检测简便。本文在双因素模型结构分析中, 是 基于数据表格 $\rightarrow$ 图表线性选择 $\rightarrow$ 模型公式程序开展的, 插 入图表进行的; 多元回归是用加载宏中的数据分析进行的, 用Exce1操作非常便捷。研究结果表明, 我们的很多实证 模型的取得多是直线性, 这违背了客观实际; 自变量解释 对因变量的作用关系, 可能存在的是曲线关系, 如对数关 系、指数关系、多次项关系等。

(2)结构转化易行。本文在转化过程中, 先由双变量数 据用计算机画出图形, 然后根据图形以 “添加趋势线” 测 试变量之间的依存关系, 再在Exce1表中, 用拖拉复制方 法计算出多次项的列变量, 最后在数据分析： “回归” 拉 入数据中, 完成多元回归结果。其原理是, 先找出双因素 模型结构, 然后变量转换, 化曲线为直线, 最终完成新的 多元线性回归, 这时的多元回归因子在包含了, 变量函数 式, 此变量属于调和变量, 本例中的时间序列变量就是如 此, 使直线性转化为混合型（既有直线又有曲线）再转化 为直线型。本文为的变量为序列变量, 引入了时间序列变 量, 并对变量关系进行不同因素关系依存处理, 使时间变 量很好的发挥了中介作用 (因变量与自变量都与之存在量 化依存关系），进而达到了模拟水平的提高。

(3)模拟水平显著。从以上变量测试转化后与转化前回 归结果比较看, 根据测试后引入的调和变量, 二元回归的 总体模拟水平

表14 二元回归模拟水平。

\begin{tabular}{llllll}
\hline RD & 0.8357 & 0.991 & 0.9962 & $+\triangle 15.53 \%$ & $+\triangle 16.05 \%$ \\
LFR & 0.8286 & 0.9949 & 0.9972 & $+\triangle 16.63$ & $+\triangle 16.76$ \\
\hline
\end{tabular}

多元回归的总体模型也有所提高

表15 多元回归模型水平。

\begin{tabular}{llll}
\hline & MLR & 二次项MLR & 三次项MLR \\
\hline RSquare & 0.996165 & 0.997949 & 0.998037 \\
AdjustedRSquare & 0.995858 & 0.997692 & 0.997695 \\
\hline
\end{tabular}

多元回归二次项的系数学生 $\mathrm{t}$ 检测, 全部达到了显著 性水平要求, 详见表 15 。

\section{2. 结论}

经过测试、变量转换, 不仅可以提高总体模拟水平, 而且还能使变量通过显著性水平检测, 据此我们有理由说,
找到了变量之间的依存关系, 找到了变量之间的依存规律, 可以根据最佳的回归模型结果去财务分析、去财务评价、 去预测决策。量化模型实证，《管理会计》、《财务分析》 和《财务管理》分别以最小二乘法或多元回归, 以及趋势 分析, 会计的预测、决策方法提及, 本文实证模型的构建, 只是事项及其指标的选择, 有悖会计本语, 但方法上, 仍 不失管理会计中预测、决策之重要方法。本文主旨在于构 建多元回归的方法技巧性，这也是基于当前实证方法泛滥 在基本的直线性模型的因素构架, 模拟水平不高, 仅满足 变量系数统计量检测的情况下通式; 本文作者认为, 社科 类建模, 虽不能像生物、生命学科那样能获得因素内生变 量, 但运用方法上的完善, 期望通过双因素变量的检测, 找出多元回归的综合最佳模型, 进而提高模型预测的精度 和科学决策的准度。限于篇幅, 本文对研究结果的应用予 以省略。

本文作者遗憾地是: 在构建多元回归时, 没有选用当 前会计核算设定的会计变量指标, 这在现行的管理学与经 济学划分细化景况下, 似有偏颇; 但可以用管理经济学交 融理解。本文有关截面数据管理会计中观、宏观模型构建 研究, 此不赘述。

\section{参考文献}

１1］张航, 刘云. 基于正相关因素混合模型结构中企业绩效评价 模型研究-来自 2015 年上市公司经验数据验证. 财务与会计 改革2016年学术研讨会 [c]. 福建省社会科学研究基地福建 江夏学院财务与会计研究中心, 教育部人文社会科学重点 研究基地厦门大学会计发展研究中心、《当代会计评论》 编辑部. 2016. 05

[2] 张航, 刘云. 截面数据引入样本序列变量对模型构建作用的 研究 [c] 中国会计学会第十五届全国会计信息化学术年会 (2016) 08

[3] 吕书龙, 刘文丽. 几类最小一乘估计回归模型的求解 [J]. 吉林师范大学学报 (自然科学版). 2011 (02)

[4] 郑箫, 金青. 回归模型与时间序列在大坝变形监测中的应用 $[J]$. 湖北师范学院学报 (自然科学版). 2010 (01)

[5] 王全众. 两类分析相关数据的Logistic 回归模型 [J]. 统计 研究. 2007 (02)

[6] 孙尚拱, 何平平. 经典的用回归模型进行统计控制中的问题 $[\mathrm{J}]$. 数理统计与管理. 2005(05)

[7] 程毛林. 两种常用的基函数回归模型 $[\mathrm{J}]$. 统计与决 策. $2002(02)$

[8] 谢远涛, 杨娟. 广义Gamma分布簇广义线性混合模型的构建 $[\mathrm{J}]$. 统计研究. $2010(10)$

[9］李春红, 张可娟, 文利霞. 基于空间自回归模型的中部经济 增长分析 $[\mathrm{J}]$. 西南大学学报 (自然科学版). 2012 (11)

[10] 陈若寒. 福建省农民收入的空间自回归模型研究 [J]. 现代 经济信息. 2013 (21) 
[11] 黄克明, 胡端平, 张国忠. 我国通货膨胀与对外经济的半相 依自回归模型的研究 [J]. 系统工程理论与实践. 2003 (04)

[12］吴国富, 孙传忠. 利用周期自回归模型对1995年部分宏观经 济指标的预测与分析 [J]. 系统工程理论与实践. 1995(07)

[13] 代洪伟, 凌能祥. 居民消费价格指数的非参数自回归模型 $[\mathrm{J}]$. 佳木斯大学学报 (自然科学版). 2012 (01)

[14] 王丽娟, 毛程连. 地方政府间土地优惠竞争关系研究一一基 于空间自回归模型的实证检验 [J]. 财经论丛. 2012 (06)

[15] 杨树旺, 冯兵. 环境库兹涅茨曲线与自回归模型用于三废污 染预测的比较分析 [J].管理世界. 2007 (03)

[16] 白仲林, 史哲. 存在测量误差的面板自回归模型的工具变量 估计 $[\mathrm{J}]$. 统计与信息论坛. 2009 (10)
[17] 胡义芳. 武汉市新建住房价格研究 [J]. 现代经济信 息. 2014(12)

[18］危黎黎, 李超, 李余辉. 基于STAR模型的人民币汇率非线性 特征及预测 $[J]$. 统计与决策. 2014 (09)

[19] Bai, J, Kao, C. "On the Estimation and Inference of a Panel Cointegration model with Cross-Sectional Dependence,". contributions to Economic Analysis

[20] Bai, J, Chihwa Kao, Serena Ng. Panel cointegration with globalstochastic trends. Journal of Econometrics. 2009 